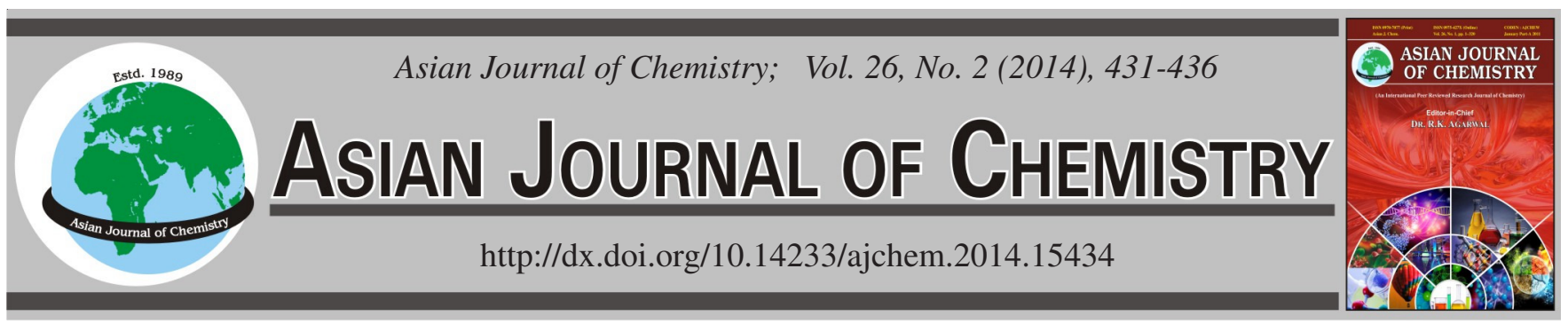

\title{
Simultaneous Determination and Pharmacokinetics of Five Furocoumarins in Rat Plasma After Oral Administration of Radix Angelicae dahuricae Extract by High Performance Liquid Chromatography
}

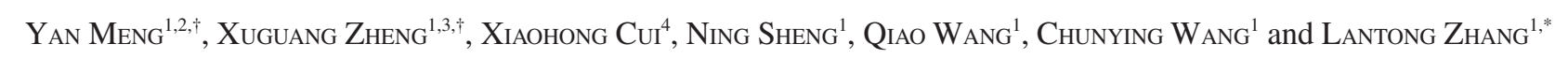

${ }^{1}$ Department of Pharmaceutical Analysis, School of Pharmacy, Hebei Medical University, Shijiazhuang 050017, P.R. China

${ }^{2}$ Department of Clinical Pharmacy, The First Hospital of Shijiazhuang, Shijiazhuang 050011, P.R. China

${ }^{3}$ Department of Pharmacy, The First Hospital of Hebei Medical University, Shijiazhuang 050031, P.R. China

${ }^{4}$ Department of Pharmacy, The Second Hospital of Hebei Medical University, Shijiazhuang 050000, P.R. China

*Corresponding author: Tel/Fax: +86 311 86266419; E-mail: zhanglantong@263.net

$\dagger$ These authors contributed equally to this work.

\begin{abstract}
Radix Angelicae dahuricae, with coumarins as its main bioactive compounds, has been widely used for antihistamine, antibacterial, spasmolysis, promote lipolysis and chronic venous insufficiency. Simultaneous determination and pharmacokinetics of coumarin compounds in rat plasma is necessary. A rapid, reliable and sensitive method for the simultaneous determination of 5 furocoumarins i.e., psoralen, bergapten, imperatorin, cnidilin and isoimperatorin in rat plasma was developed using high-performance liquid chromatographic. The established method could be easily applied to the determination in vivo and pharmacokinetic studies of five coumarin compounds in rat plasma after oral administration of Radix Angelicae Dahuricae extract.
\end{abstract}

Keywords: Radix Angelicae dahuricae, Coumarins, HPLC-DAD, Pharmacokinetics.

ᄂ - - - - - - - - - - - - - - - - - - - - - - - - - -

\section{INTRODUCTION}

Radix Angelicae dahuricae, the dried radix of Angelica dahurica (Fisch. ex Hoffm.) Benth. et Hook. f. Angelica dahurica (Fisch. ex Hoffm.) Benth. et Hook. f. var. formosana (Boiss.) Shan et Yuan, is a well known traditional Chinese medicine (TCM). It has been widely used in China for treatment of colds, headaches, toothache, coryza, vitiligo, trauma and leukorrhea, etc. ${ }^{1}$.

As reported previously, the chemical composition of Radix Angelicae dahuricae mainly includes aetherolea, coumarins and glycoside, among which coumarins are generally considered as the major components, such as psoralen, bergapten, imperatorin, cnidilin and isoimperatorin, etc. ${ }^{2,3}$. Until now pharmacological studies and clinical practice demonstrated that the major active compounds of Radix Angelicae dahuricae were coumarin compounds, which had remarkable antihistamine ${ }^{4}$, antibacterial ${ }^{5,6}$, spasmolysis ${ }^{7}$, Promote lipolysis ${ }^{8}$ and chronic venous insufficiency ${ }^{9}$.

To date, there have already been many preliminary researches about the quantitative analysis of bergapten, imperatorin, cnidilin and/or isoimperatorin in plant material, natural product and preparations ${ }^{10,11}$. In contrast, only three papers have been reported for the pharmacokinetic studies of imperatorin in rat by HPLC, oxypeucedanin hydrate and byakangelicin in dog by HPLC and isoimperatorin in rat by GC$\mathrm{MS}$, independently ${ }^{12-14}$. As psoralen, bergapten, imperatorin, cnidilin and isoimperatorin are the major active components in Radix Angelicae dahuricae, it is essential to develop a method to determine them in plasma samples for the purpose of studying the mechanism of action. However, to our best of knowledge, there has been no method established for the simultaneous determination of the five active constituents for pharmacokinetic investigations.

The purpose of the present work was to develop and validate a simple, fast and reliable RP- HPLC method with DAD detection for the determination of psoralen, bergapten, imperatorin, cnidilin and isoimperatorin in rat plasma. The results indicated that five coumarin compounds could be quantified simultaneously and the method was successful for application of studies on pharmacokinetics of the five components.

\section{EXPERIMENTAL}

The dried of Radix Angelicae dahuricae purchased from An-guo (Hebei, China) was grounded to powder and then passed through a 60-mesh sieve for preparation. The psoralen, 
bergapten, imperatorin, cnidilin and isoimperatorin were isolated from Radix Angelicae dahuricae in our laboratory. All these compounds were identified by direct comparison of their ${ }^{1} \mathrm{H}$ NMR, ${ }^{13} \mathrm{C}$ NMR and MS spectral data with those reported in the literature ${ }^{15,16}$ and their purities were no less than $98 \%$ by HPLC analysis. Acetonitrile and methanol were of HPLC grade and obtained from Tedia (Tedia, Fairfield, USA). The distilled water was prepared from demineralized water and used throughout the study. All other chemical solvents were of analytical grade from Beijing Chemical Factory (Beijing, China).

Instrument and chromatographic conditions: Experiments was performed on the Agilent 1200 series HPLC system (Agilent, USA) consisting of a quaternary pump, an autosampler, a degasser, an automatic thermostatic column compartment and controlled by the chemstation software (Agilent). The analytical column used was a Waters SunFire ${ }^{\mathrm{TM}} \mathrm{C}_{18}$ column $(150 \mathrm{~mm} \times 4.6 \mathrm{~mm}$ i.d., $5 \mu \mathrm{m})$ for ultimate performance. The mobile phase for HPLC analysis consisted of acetonitrile-water using gradient elution (0-5 min, 40-45\% acetonitrile; $20 \mathrm{~min}$, $80 \%$ acetonitrile). The flow rate was $1 \mathrm{~mL} / \mathrm{min}$ and the column temperature was $30^{\circ} \mathrm{C}$. The detection wavelength was set at $310 \mathrm{~nm}$ and the sample injection volume was $60 \mu \mathrm{L}$. The peak identification was based on the retention time and the DAD spectrum against the standards presented in the chromatogram. Their chemical structures are shown in Fig. 1.

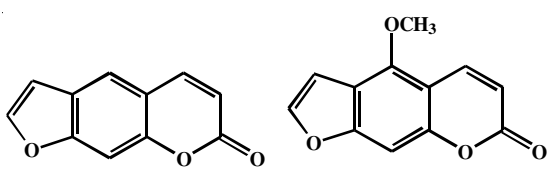

(1) Psoralen

(2) Bergapten

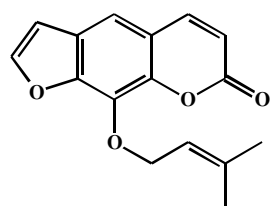

(3) Imperatorin

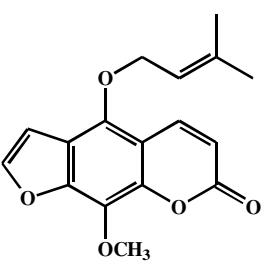

(4) Cnidilin

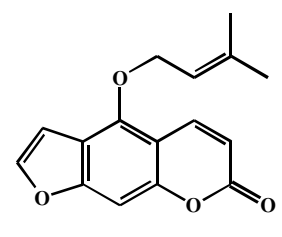

(5) Isoimperatorin
Fig. 1. Chemical structures of five Coumarin Compounds: (1) psoralen; (2) Bergapten; (3) Imperatorin; (4) Cnidilin; (5) Isoimperatorin

Preparation of the standard solutions: Stock solutions containing psoralen, bergapten, imperatorin, cnidilin and isoimperatorin were prepared by dissolving in methanol to yield concentrations of 868.0, 935.2, 2496.0, 1600.0 and $1416.8 \mathrm{ng} / \mathrm{mL}$ for each analyte, respectively.

All stock solutions were further diluted to give a series of concentrations for plasma calibration standards (26.0, 156.2, $312.5,468.7,625.0,781.2$ and $833.3 \mathrm{ng} / \mathrm{mL}$ for psoralen; 28.1 , 168.3, 336.7, 505.0, 673.3, 841.7 and $897.8 \mathrm{ng} / \mathrm{mL}$ for bergapten; 74.9, 449.3, 898.6, 1347.8, 1797.1, 2246.4 and $2396.2 \mathrm{ng} / \mathrm{mL}$ for imperatorin; 48, 288, 576, 864, 1152, 1440 and $1563 \mathrm{ng} / \mathrm{mL}$ for cnidilin and 42.5, 255.0, 510.1, 765.1, 1020.1, 1275.1 and $1360.1 \mathrm{ng} / \mathrm{mL}$ for isoimperatorin). The quality control (QC) samples were prepared in the same way as the calibration samples to make low, medium and high concentrations $(26,312.5$ and $781.2 \mathrm{ng} / \mathrm{mL}$ for psoralen; 28.1, 336.7 and $841.7 \mathrm{ng} / \mathrm{mL}$ for bergapten; 74.9, 898.6 and $2246.4 \mathrm{ng} / \mathrm{mL}$ for imperatorin; 48 , 576 and $1440 \mathrm{ng} / \mathrm{mL}$ for cnidilin and 42.5, 510.1 and 1275.1 $\mathrm{ng} / \mathrm{mL}$ for isoimperatorin).

Preparation of Angelica dahurica extract: Radix Angelicae dahuricae $(1000 \mathrm{~g})$ was extracted thrice with $95 \%$ ethanol (10 L), $1 \mathrm{~h}$ for each time. The extracted solutions were combined and ethanol was removed under reduced pressure and then the residue was suspended in water and partitioned three times with petroleum ether and ethyl acetate, combined ethyl acetate and then removed it under reduced pressure. The residue was coarsely eluted by column chromatography on silica gel (A, petroleum ether-ethyl acetate, 5:1; B, petroleum ether-ethyl acetate, 4:1; C, petroleum ether-ethyl acetate $3: 1$; D, petroleum ether-ethyl acetate, 2:1; E, petroleum ether-ethyl acetate, 1:1), Each collection elution was analyzed by TLC. Consequently, the Fr.B was used to yield active components. The fraction was evaporated to dryness under reduced pressure, the residue was suspended in $0.5 \%$ carboxymethylcellulose sodium. To calculate the administered dose, the contents of five coumarin compounds in administration solution were quantitatively determined by external standardization method using the same chromatography conditions as described above. The contents of psoralen, bergapten, imperatorin, cnidilin, isoimperatorin were $0.47,0.35,4.22,3.00$ and $2.38 \mathrm{mg} / \mathrm{mL}$, respectively.

Plasma sample preparation: $100 \mu \mathrm{L}$ plasma sample was added to $300 \mu \mathrm{L}$ methanol in a centrifuge tube. The mixture was vortexed for $1 \mathrm{~min}$ and centrifuged at $12,000 \times \mathrm{g}$ for 10 min to separate the protein from the organic phase, $60 \mu \mathrm{L}$ of the supernatant was injected into the HPLC system for analysis.

Selectivity: Selectivity was investigated by comparing chromatograms of blank plasmas obtained from six rats with those of corresponding standard plasma samples spiked with psoralen, bergapten, imperatorin, cnidilin and isoimperatorin and plasma samples after oral administration of Angelica dahurica extract. All six blank plasma samples were extracted to ensure the absence of interfering peaks.

Linearity of calibration curve and lower limit of quantification (LLOQ): The control plasma samples spiked with psoralen, bergapten, imperatorin, cnidilin and isoimperatorin at seven concentrations over the ranges of 26.0-833.3, 28.1897.8, 74.9-2396.2, 48.0-1536.1 and 45.5-1360.1 ng/mL. Calibration curves for the five compounds in plasma were generated by plotting the peak area $(\mathrm{Y})$ versus those nominal concentrations $(\mathrm{X})$ in standard plasma by the $1 / \mathrm{x}^{2}$ weighted least-square linear regression. The LLOQ was defined as the lowest drug concentration on the plasma and determined at a signal-to-noise ratio of 5:1.

Precision and accuracy: The intra-day precision and accuracy were determined for each compound by analyzing five replicates quality control samples (low, medium and high concentrations) in a single day, whilst the inter-day values were carried out over three consecutive days. The concentration of each sample was determined using a calibration curve prepared on the same day. The intra-day and inter-day precision was defined as RSD and the accuracy was assessed by comparing the measured concentration with its true value. 
Extraction recovery: Recoveries of psoralen, bergapten, imperatorin, cnidilin and isoimperatorin were evaluated at three concentrations levels of quality control samples on the same day. Recovery data were determined by comparing the peak area ( $\mathrm{n}=5$ at each concentration) obtained from plasma samples spiked with analyte before extraction with those from the corresponding standards diluted with water.

Stability: To evaluate sample stability after three freezethaw cycles and at room temperature, three replicates of quality control samples were subjected to three freeze-thaw cycles or were processed and stored under autosampler conditions for $24 \mathrm{~h}$. Stability was assessed by comparing the mean concentration of the stored quality control samples with the mean concentration of freshly prepared quality control samples.

Application of the method: Male Sprague-Dawley rats $(250 \pm 20 \mathrm{~g})$ were supplied by Lab Animal Center of Heibei Medical University (Shijiazhuang, China). They were kept in environmentally controlled breeding room for 4 days until the time of the experiment. They were fed with standard laboratory food and water.

After being fasted for $12 \mathrm{~h}$ with free access to water, Radix Angelicae dahuricae extract was orally administered to the rats at a dose of $4 \mathrm{~mL} / \mathrm{kg}$ (equivalent to $1.88 \mathrm{mg} / \mathrm{kg}$ of psoralen, $1.4 \mathrm{mg} / \mathrm{kg}$ of bergapten, $16.88 \mathrm{mg} / \mathrm{kg}$ of imperatorin, $12 \mathrm{mg} / \mathrm{kg}$ of cnidilin and $9.52 \mathrm{mg} / \mathrm{kg}$ of isoimperatorin). Blood samples $(0.50 \mathrm{~mL})$ were collected into heparinized centrifuge tubes from the via fossa orbitalis vein at $0.5,1,2,3,4,5,6,7$, $8,10,15,24 \mathrm{~h}$ and then immediately centrifuged at $4,000 \times \mathrm{g}$ for $10 \mathrm{~min}$. The separated plasma was frozen at $-20^{\circ} \mathrm{C}$ prior to analysis.

Pharmacokinetic data analysis: Pharmacokinetic analysis of concentration-time data was performed using Excel software. The pharmacokinetic parameters, such as maximum plasma concentration $\left(\mathrm{C}_{\max }\right)$ and time of maximum concentration $\left(\mathrm{T}_{\max }\right)$, were obtained directly from the plasma concentrationtime plots. The elimination rate constants $(\mathrm{k})$ were determined by linear regression on the logarithmic transformation of the last four data points of the curve. The elimination half-life $\left(T_{1 / 2}\right)$ was calculated by the following equation: $T_{1 / 2}=0.693 / \mathrm{k}$. The area under the plasma concentration versus time curve up to the last time $(\mathrm{t})\left(\mathrm{AUC}_{0-\mathrm{t})}\right.$ was determined using the trapezoidal rule. The $\mathrm{AUC}_{0-\infty}$ values were calculated by adding the value of $\mathrm{C}_{\mathrm{t}} \times \mathrm{k}^{-1}$ to $\mathrm{AUC}_{0-\mathrm{t}}$.

\section{RESULTS AND DISCUSSION}

Preparation of plasma: Commonly, the used extraction techniques mainly include liquid-liquid extraction, protein precipitation and solid phase extraction (SPE). In the course of our method development, we found that liquid-liquid extraction technique had a low recovery when ethyl acetate or $n$-butanol used as extraction solvents. The solid phase extraction has good extraction recovery and reproducibility, however, this extraction method is expensive. While, acceptable recovery for five analyties, clear supernatant and good separation from interfering peaks were obtained by using methanol as the plasma protein precipitating reagent. Therefore, plasma samples were subjected to a simple protein precipitation procedure. The present method considerably reducing the sample processing time is rapid, simple without any loss of analytes.
Selectivity and chromatography: The specificity of the method was determined by comparing the chromatograms of blank plasmas with the corresponding spiked plasma and plasma sample. Typical chromatograms of a blank plasma sample, a spiked plasma standard and plasma samples from a rat at $4 \mathrm{~h}$ after oral administration are shown in Fig. 2. No interferences from endogenous substances were observed at the retention time regions of the analyte. The retention times of psoralen, bergapten, imperatorin, cnidilin and isoimperatorin were 5.8, 7.6, 13.4, 14.4 and $15.5 \mathrm{~min}$, respectively. The analytes were well separated from co-extracted materials under the described chromatographic conditions.
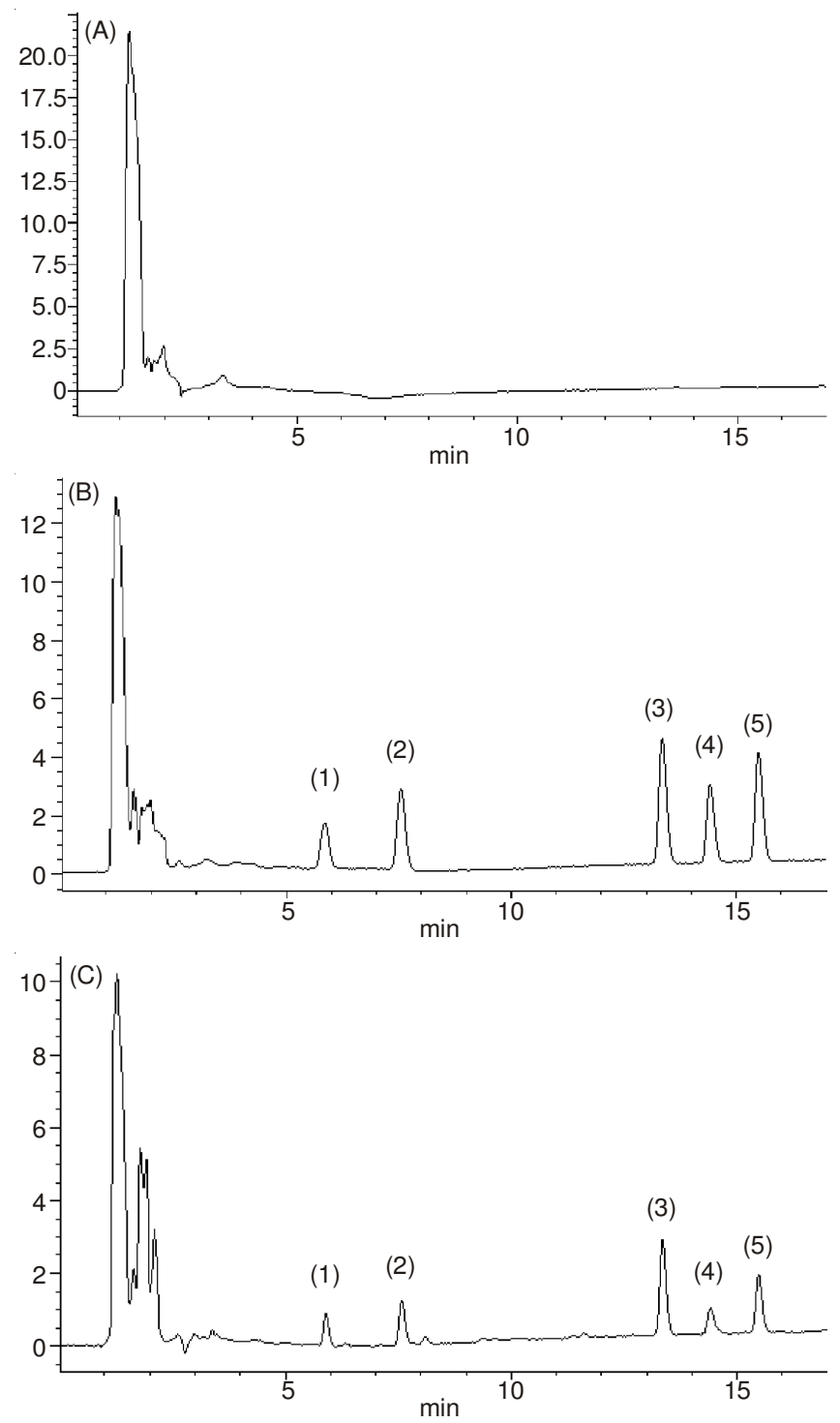

Fig. 2. Chromatograms of rat plasma samples: (A) blank plasma; (B) plasma spiked with psoralen (1), bergapten (2), imperatorin (3), cnidilin (4) and isoimperatorin (5); (C) plasma sample after oral administration of Radix Angelicae dahuricae extract

Linearity and sensitivity: The standard curves of the peak area $(\mathrm{Y})$ to the concentration $(\mathrm{X})$ were constructed using $1 / \mathrm{x}^{2}$ weighted linear least-aquares regression model. The standard calibration for psoralen, bergapten, imperatorin, cnidilin and isoimperatorin were linear over the ranges of 26.04-833.28, 28.06-897.80, 74.88-2396.16, 48.00-1536.12 and 45.50- 


\begin{tabular}{|c|c|c|c|c|c|c|}
\hline \multirow{2}{*}{$\begin{array}{c}\text { Spiked } \\
\text { concentratin }(\mathrm{ng} / \mathrm{mL})\end{array}$} & \multicolumn{3}{|c|}{ Intra-day $(n=5)$} & \multicolumn{3}{|c|}{ Inter-day $(n=3)$} \\
\hline & $\begin{array}{l}\text { Measured concentration } \\
(\mathrm{ng} / \mathrm{mL}, \text { mean } \pm \mathrm{SD})\end{array}$ & $\mathrm{RSD}(\%)$ & $\begin{array}{c}\text { Accuracy } \\
(\%)\end{array}$ & $\begin{array}{l}\text { Measured concentration }(\mathrm{ng} / \mathrm{mL}, \\
\text { mean } \pm \mathrm{SD})\end{array}$ & $\begin{array}{c}\text { RSD } \\
(\%)\end{array}$ & $\begin{array}{c}\text { Accuracy } \\
(\%)\end{array}$ \\
\hline \multicolumn{7}{|c|}{ Psoralen } \\
\hline 26.0 & $27.7 \pm 4.1$ & 14.8 & 106.4 & $29.1 \pm 4.2$ & 14.5 & 111.8 \\
\hline 312.5 & $337.9 \pm 38.5$ & 11.4 & 108.1 & $342.4 \pm 15.4$ & 4.5 & 109.6 \\
\hline 781.2 & $823.4 \pm 33.3$ & 4.1 & 105.4 & $794.3 \pm 2.2$ & 0.3 & 101.7 \\
\hline \multicolumn{7}{|c|}{ Bergapten } \\
\hline 28.1 & $31.4 \pm 4.6$ & 14.7 & 111.7 & $27.3 \pm 2.7$ & 9.8 & 97.2 \\
\hline 336.7 & $354.6 \pm 45.7$ & 12.9 & 105.3 & $363.1 \pm 45.6$ & 12.6 & 107.9 \\
\hline 841.7 & $858.4 \pm 30.6$ & 3.6 & 102.0 & $869.8 \pm 32.2$ & 3.7 & 103.3 \\
\hline \multicolumn{7}{|c|}{ Imperatorin } \\
\hline 74.9 & $85.8 \pm 11.9$ & 13.9 & 114.6 & $83.3 \pm 10.54$ & 12.7 & 111.2 \\
\hline 898.6 & $1005.1 \pm 96.8$ & 9.6 & 111.9 & $869.1 \pm 57.7$ & 6.6 & 96.7 \\
\hline 2246.4 & $2396.9 \pm 64.5$ & 2.7 & 106.7 & $2321.3 \pm 30.1$ & 1.3 & 103.3 \\
\hline \multicolumn{7}{|c|}{ Cnidilin } \\
\hline 48.0 & $53.8 \pm 5.9$ & 10.9 & 112.0 & $50.6 \pm 2.6$ & 5.2 & 105.4 \\
\hline 576.0 & $636.5 \pm 49.2$ & 7.7 & 110.5 & $559.3 \pm 39.3$ & 7.0 & 97.1 \\
\hline 1440.0 & $1552.5 \pm 55.3$ & 3.6 & 107.8 & $1544.5 \pm 102.1$ & 6.6 & 107.3 \\
\hline \multicolumn{7}{|c|}{ Isoimperatorin } \\
\hline 45.5 & $50.3 \pm 6.1$ & 12.2 & 110.4 & $45.9 \pm 6.6$ & 14.4 & 101.0 \\
\hline 510.1 & $582.3 \pm 24.0$ & 4.1 & 114.2 & $516.3 \pm 59.6$ & 11.6 & 101.2 \\
\hline 1275.1 & $1359.8 \pm 55.9$ & 4.1 & 106.6 & $1304.4 \pm 1.7$ & 0.1 & 102.3 \\
\hline
\end{tabular}

$1360.12 \mathrm{ng} / \mathrm{mL}$ in rat plasma, respectively. The mean values of regression equation of the analytes in rat plasma were $\mathrm{Y}=$ $0.0321 \mathrm{X}-0.0474(\mathrm{r}=0.9981$, psoralen $), \mathrm{Y}=0.0527 \mathrm{X}+0.5625$ ( $\mathrm{r}=0.9960$, bergapten), $\mathrm{Y}=0.0282 \mathrm{X}-0.0594(\mathrm{r}=0.9977$, imperatorin), $\mathrm{Y}=0.027 \mathrm{X}+0.3487(\mathrm{r}=0.9983$, cnidilin $)$ and $\mathrm{Y}=0.0427 \mathrm{X}+0.0542(\mathrm{r}=0.9979$, isoimperatorin $)$. The LOQ were found to be $20.22 \mathrm{ng} / \mathrm{mL}$ for psoralen, $24.31 \mathrm{ng} / \mathrm{mL}$ for bergapten, $51.25 \mathrm{ng} / \mathrm{mL}$ for imperatorin, $24.57 \mathrm{ng} / \mathrm{mL}$ for cnidilin and $21.02 \mathrm{ng} / \mathrm{mL}$ for isoimperatorin in rat plasma in rat plasma, respectively. The LOD were found to be $10.41 \mathrm{ng} / \mathrm{mL}$ for psoralen, $11.22 \mathrm{ng} / \mathrm{mL}$ for bergapten, $29.95 \mathrm{ng} / \mathrm{mL}$ for imperatorin, $15.20 \mathrm{ng} / \mathrm{mL}$ for cnidilin and $12.01 \mathrm{ng} / \mathrm{mL}$ for isoimperatorin in rat plasma, respectively.

Precision and accuracy: The precision and accuracy of the assay were evaluated with quality control samples at low, medium and high concentrations. Results are summarized in Table-1. The intra-day and inter-day precisions calculated as the relative standard deviation (RSD) were $<15 \%$. The accuracies of five coumarin compounds were within the range of 97.1-114.6\%.

Extraction recovery: The mean extraction recoveries and RSD for the five coumarin compounds from rat plasma were assessed at low, medium and high concentrations. All recoveries were within the range of 87.1-109.2\% (Table-2).

Stability: The results of storage stability in rat plasma are shown in Table-3. The results showed that five analytes were stable for $24 \mathrm{~h}$ in autosampler conditions and in plasma samples following three freeze-thaw cycles.

Application of analytical method in pharmacokinetic studies: The validated method was successfully applied to monitor the concentrations and pharmacokinetic studies of five coumarin compounds in rat plasma after a single oral administration of Radix Angelicae dahuricae extract with a dosage of $4 \mathrm{~mL} / \mathrm{kg}$. The mean plasma concentration-time curves $(n=6)$ of psoralen, bergapten, imperatorin, cnidilin and isoimperatorin

\begin{tabular}{cccc}
\multicolumn{4}{c}{ TABLE-2 } \\
RECOVERY OF COUMARIN COMPOUNDS \\
FROM RAT PLASMA $(\mathrm{n}=6)$
\end{tabular}

were shown in Fig. 3. The pharmacokinetic parameters are listed in Table-4.

In general, half-life $\left(\mathrm{T}_{1 / 2}\right)$ values for five coumarin compounds were 8.57, 6.13, 6.47, 6.86 and $6.91 \mathrm{~h}$, respectively. The four coumarin compounds (bergapten, imperatorin, cnidilin and isoimperatorin) carried similar $\mathrm{T}_{1 / 2}(c a .6 \mathrm{~h})$ while psoralen had longer $\mathrm{T}_{1 / 2}(\mathrm{ca} .8 \mathrm{~h})$, which might be caused by the side chain. In the chemical structures of the latter compounds, there were side chain to link but psoralen did not have. Although the doses of the five coumarin compounds given to animals were different for Radix Angelicae dahuricae extract (1.88 mg/kg of psoralen, $1.40 \mathrm{mg} / \mathrm{kg}$ of bergapten, $16.88 \mathrm{mg} /$ $\mathrm{kg}$ of imperatorin, $12.00 \mathrm{mg} / \mathrm{kg}$ of cnidilin and $9.52 \mathrm{mg} / \mathrm{kg}$ of isoimperatorin), the AUC values got from the pharmacokinetic parameters were not in accordance to the proportion of the dose. The AUC value of psoralen was $4791.8 \mathrm{ng} \mathrm{h} / \mathrm{mL}$ at dose of $1.88 \mathrm{mg} / \mathrm{kg}$ while cnidilin was only $4210.0 \mathrm{ng} \mathrm{h} / \mathrm{mL}$ at dose 


\begin{tabular}{|c|c|c|c|c|c|c|}
\hline \multirow{3}{*}{$\begin{array}{c}\text { Spiked } \\
\text { concentration } \\
(\mathrm{ng} / \mathrm{mL})\end{array}$} & \multicolumn{5}{|c|}{$\begin{array}{c}\text { TABLE-3 } \\
\text { STABILITY OF COUMARIN COMPOUNDS IN RAT PLASMA }\end{array}$} & \\
\hline & \multicolumn{3}{|c|}{ Freeze-thaw cycles } & \multicolumn{3}{|c|}{$24 \mathrm{~h}$ in autosampler conditions } \\
\hline & $\begin{array}{l}\text { Freshly prepared } \\
\text { samples (ng/mL) }\end{array}$ & $\begin{array}{l}\text { Stored samples } \\
(\mathrm{ng} / \mathrm{mL})\end{array}$ & Accuracy $(\%)$ & $\begin{array}{l}\text { Freshly prepared } \\
\text { samples (ng/mL). }\end{array}$ & $\begin{array}{l}\text { Stored samples } \\
(\mathrm{ng} / \mathrm{mL})\end{array}$ & Accuracy $(\%)$ \\
\hline \multicolumn{7}{|c|}{ Psoralen } \\
\hline 26.0 & 27.5 & 28.3 & 103.2 & 29.3 & 29.4 & 100.4 \\
\hline 312.5 & 319.2 & 325.5 & 102.0 & 341.5 & 351.9 & 103.0 \\
\hline 781.2 & 817.7 & 808.3 & 98.9 & 861.3 & 820.5 & 95.3 \\
\hline \multicolumn{7}{|c|}{ Bergapten } \\
\hline 28.1 & 29.2 & 27.6 & 94.5 & 33.0 & 30.8 & 93.3 \\
\hline 336.7 & 300.5 & 319.5 & 106.3 & 385.9 & 322.0 & 83.4 \\
\hline 841.7 & 826.1 & 845.1 & 102.3 & 841.3 & 879.3 & 104.5 \\
\hline \multicolumn{7}{|c|}{ Imperatorin } \\
\hline 74.9 & 80.3 & 73.0 & 90.9 & 80.4 & 90.9 & 113.0 \\
\hline 898.6 & 948.9 & 1034.0 & 109.0 & 990.8 & 1036.9 & 104.7 \\
\hline 2246.4 & 2463.1 & 2491.5 & 101.2 & 2370.9 & 2304.5 & 97.2 \\
\hline \multicolumn{7}{|c|}{ Cnidilin } \\
\hline 48.0 & 50.1 & 46.4 & 92.6 & 57.5 & 53.8 & 93.6 \\
\hline 576.0 & 598.2 & 624.1 & 104.3 & 607.5 & 653.4 & 107.6 \\
\hline 1440.0 & 1590.8 & 1520.4 & 95.6 & 1538.9 & 1535.2 & 99.8 \\
\hline \multicolumn{7}{|c|}{ Isoimperatorin } \\
\hline 45.5 & 47.6 & 51.9 & 109.1 & 50.3 & 55.0 & 109.3 \\
\hline 510.1 & 579.5 & 574.8 & 99.2 & 560.0 & 512.3 & 91.5 \\
\hline 1275.1 & 1401.2 & 1443.7 & 103.0 & 1343.0 & 1357.0 & 101.1 \\
\hline
\end{tabular}

\begin{tabular}{cccccc} 
& \multicolumn{5}{c}{ TABLE-4 } \\
& \multicolumn{5}{c}{$\begin{array}{c}\text { PHARMACOKINETIC PARAMETERS OF FIVE COUMARIN COMPOUNDS AFTER } \\
\text { ORAL ADMINISTRATION OF Radix Angelicae Dahuricae EXTRACT IN RAT. }(\mathrm{n}=6)\end{array}$} \\
\cline { 2 - 6 } Pharmacokinetic & \multicolumn{5}{c}{ Mean \pm SD } \\
parameters & Psoralen & Bergapten & Imperatorin & Cnidilin & Isoimperatorin \\
\cline { 2 - 6 } & $811.4 \pm 41.6$ & $588.9 \pm 31.8$ & $2070.2 \pm 311.0$ & $753.8 \pm 221.6$ & $870.9 \pm 249.5$ \\
$\mathrm{C}_{\max }(\mathrm{ng} / \mathrm{mL})$ & $4.0 \pm 0.0$ & $4.0 \pm 0.0$ & $5.00 \pm 0.00$ & $5.0 \pm 0.0$ & $5.0 \pm 0.0$ \\
$\mathrm{~T}_{\max }(\mathrm{h})$ & $8.57 \pm 1.54$ & $6.13 \pm 0.96$ & $6.47 \pm 1.16$ & $6.86 \pm 0.56$ & $6.91 \pm 0.77$ \\
$\mathrm{~T}_{1 / 2}(\mathrm{~h})$ & $0.0809 \pm 0.0255$ & $0.1130 \pm 0.0182$ & $0.1070 \pm 0.0227$ & $0.1010 \pm 0.0094$ & $0.1003 \pm 0.0208$ \\
$\mathrm{k}(1 / \mathrm{h})$ & $4157.4 \pm 317.6$ & $2777.6 \pm 455.8$ & $11322.1 \pm 1150.8$ & $3948.5 \pm 498.8$ & $5110.8 \pm 1107.2$ \\
$\mathrm{AUC}_{0-\mathrm{t}}(\mathrm{ng} \mathrm{h} / \mathrm{mL})$ & $4791.8 \pm 347.7$ & $2918.3 \pm 522.8$ & $12037.4 \pm 1122.4$ & $4210.0 \pm 497.0$ & $5323.6 \pm 1144.6$ \\
$\mathrm{AUC}_{0-\infty}(\mathrm{ng} \mathrm{h} / \mathrm{mL})$ & & & & &
\end{tabular}

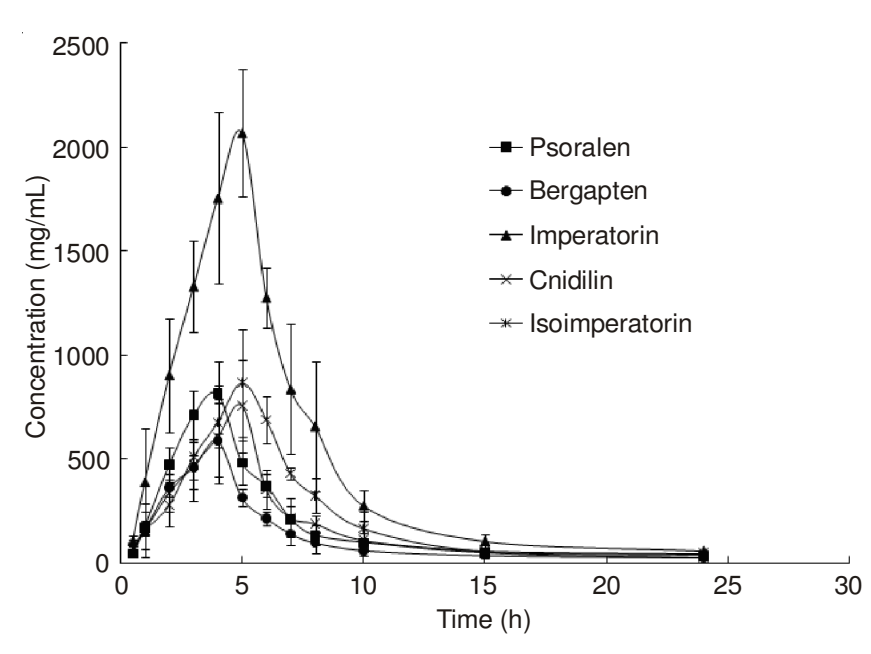

Fig. 3. Mean plasma concentration-time plots of psoralen, bergapten, imperatorin, cnidilin and isoimperatorin after a single oral dose of Radix Angelicae dahuricae extract to rats

of $12 \mathrm{mg} / \mathrm{kg}$. The dose of imperatorin was the highest, the amounts were 9.0, 12.1, 1.4 and 1.8 times than the dose of psoralen, bergapten, cnidilin and isoimperatorin, respectively. But The AUC values were 2.5, 4.1, 2.9 and 2.3 times, respectively. Compared by the amount of the 5 coumarin compounds, psoralen and bergapten had much more bioavailability. Form this paper, it showed that the total results of the pharmacokinetic behaviors of psoralen, bergapten, imperatorin, cnidilin and isoimperatorin in rats after oral administration of Radix Angelicae dahuricae extract were similar, owing to their similar chemical structures. The results also were similar with that in the reference ${ }^{12}$.

\section{Conclusion}

The present study described a simple and reliable HPLCDAD method for the simultaneous determination of five coumarin compounds in blood samples. This is the first validated HPLC method for simultaneous quantification of psoralen, bergapten, imperatorin, cnidilin and isoimperatorin in rat plasma, which has good recovery, sensitivity and reproducibility. It was successfully applied to the pharmacokinetic studies of active components of Radix Angelicae dahuricae, which will play an important role in investigating the action mechanism of Radix Angelicae dahuricae.

\section{ACKNOWLEDGEMENTS}

The authors thank the financial support from the Natural Science Foundation of Hebei Province-CSPC Pharmaceutical Joint Research Fund, China (H2012206079). 


\section{REFERENCES}

1. The Pharmacopoeia of the People's Republic of China, The Pharmacopoeia Commission of PRC, Part I, Beijing (2005).

2. X.L. Piao, I.H. Park, S.H. Baek, H.Y. Kim, M.K. Park and J.H. Park, J. Ethnopharmacol., 93, 243 (2004).

3. Y. Saiki, K. Morinaga, O. Okegawa, S. Sakai and Y. Amaya, Yakugaku Zasshi, 91, 1313 (1971).

4. Y. Kimura, H. Okuda and K. Baba, J. Nat. Prod., 60, 249 (1997).

5. Y.S. Kwon, A. Kobayashi, S. Kajiyama, K. Kawazu, H. Kanzaki and C.M. Kim, Phytochemistry, 44, 887 (1997).

6. F. Cottiglia, G. Loy, D. Garau, C. Floris, M. Casu, R. Pompei and L. Bonsignore, Phytomedicine, 8, 302 (2001).

7. S.X. Deng and Y.Q. Mo, Chin. Trad. Herbal Drugs, 19, 22 (1988).

8. Y. Kimura, H. Ohminami, H. Arichi, H. Okuda, K. Baba, M. Kozawa and S. Arichi, Planta Med., 45, 183 (1982).
9. B.S. Adam, R. Pentz, C.P. Siegers, O. Strubelt and M. Tegtmeier, Phytomedicine, 12, 52 (2005).

10. T.T. Wang, H. Jin, Q. Li, W.M. Cheng, Q.Q. Hu, X.H. Chen and K.S. Bi, Chromatographia, 65, 477 (2007)

11. J.Y. Deng, G.H. Gao, C.J. Zhao and P.J. Xu, J. Shenyang Pharm. Univ., 21, 354 (2004).

12. Y.B. Li, J. Pharm. Biomed. Anal., 40, 1253 (2006).

13. Y. Xie, Y. Chen, M. Lin, J. Wen, G.R. Fan and Y.T. Wu, J. Pharm. Biomed. Anal., 44, 166 (2007).

14. S.C. Wang, Q.H. Chen and L.C. He, J. Chromatogr. B, 852, 473 (2007).

15. E.S. Waight, T.K. Razdan, B. Qadri and S. Harkar, Phytochemistery, 26, 2063 (1987).

16. S. Harkar, T.K. Razdan and E.S. Waight, Phytochemistery, 23, 419 (1984). 